\title{
HIV Preexposure Prophylaxis Training in Family Medicine Residencies: A National Survey
}

Byron K. Jasper, MD, MPH; James N. Becker, MD; Allison E. Myers, MD, MPH;

Peter F. Cronholm, MD, MSCE

BACKGROUND AND OBJECTIVES: Preexposure prophylaxis (PrEP) reduces HIV transmission among high-risk individuals. Yet, the HIV epidemic continues to expand among marginalized populations and America's Southeastern states. Various barriers remain to PrEP uptake, namely provider knowledge and education. We sought to investigate residency training, competency, and prescribing of PrEP among population size. Additionally, we asked program directors to identify barriers to PrEP.

METHODS: We surveyed family medicine program directors as part of the Council of Academic Family Medicine Educational Research Alliance survey from January 2018 through February 2018.

RESULTS: Our survey questions had a $52.9 \%$ (276/522) response rate. No programs in rural communities less than 30,000 population $(0 / 27)$ reported significant PrEP training for their residents; those in nonrural communities of at least 30,000 reported this training more frequently $(41 / 246,16.7 \%)$. Compared to Fischer expected values, the finding was statistically significant $(P=.019)$; using a 75,000 population demarcation lowered significance $(P=.192)$. We found programs that identify significant PrEP training also cite more PrEP prescribing within their practice (OR 7.27, $P<.001)$. Programs with significant training also report their residents graduate with greater PrEP competency (OR $18.33, P<.001)$. The largest barriers identified were faculty expertise, not having enough high-risk patients, inadequate screening, and resident knowledge/ training.

CONCLUSIONS: We identified natural associations between increased training in PrEP and perceived PrEP competencies. We identified a lack of significant PrEP training and associated PrEP competencies in rural residency programs. Barriers identified in this study can help inform curricular needs to improve primary care workforce capacity to lower HIV risk.

(Fam Med. 2022;54(1):24-9.)

doi: 10.22454/FamMed.2022.740210

n 2012, the Food and Drug Administration approved the use of emtricitabine/tenofovir disoproxil fumarate (Truvada) as preexposure prophylaxis (PrEP) for HIV-negative individuals. Since that time, emtricitabine/tenofovir alefenamide (Descovy) has been approved for HIV-negative men and transgender trained in its use and willing to prescribe PrEP. Primary care providers (PCPs) should be equally, if not more, knowledgeable of the utility of PrEP and practiced in its delivery within primary care settings, as many HIV specialists may not care for a large number of patients who are HIV negative. Furthermore, patients in rural areas often lack access to this preventative treatment due to barriers that include large distances to nearest PrEP provider, inadequate funding to support PrEP distribution in those areas, and insufficient telehealth resources within those communities. ${ }^{9-10}$

Access to PrEP requires health care providers to be aware of its existence, competent to discuss and prescribe it for appropriate patient populations, or comfortable to refer patients elsewhere. Many of the studies on use of PrEP have evaluated potential barriers for providers. One study identified that the HIV providers who have the knowledge and skills necessary to prescribe PrEP may see few HIV-negative individuals. ${ }^{11}$ In contrast, PCPs may

From Byja Health Systems, Inc, Baton Rouge, LA (Dr Jasper); Department of Family and Community Medicine, ChristianaCare Health System, Wilmington, DE (Dr Becker); MetroHealth Department of Family Medicine, Case Western Reserve University School of Medicine, Cleveland, $\mathrm{OH}$ (Dr Myers); and Department of Family Medicine and Community Health, Center for Public Health initiatives, Leonard Davis Institute of Health Economics, University of Pennsylvania, Philadelphia, PA (Dr Cronholm). 
encounter high-risk HIV-negative patients, but may be unaware of PrEP or uncomfortable prescribing it. Other study participants have also referred to concerns about effectiveness of PrEP in real-world settings, potential risk disinhibition, medication toxicity, and antiretroviral drugresistance induction. ${ }^{12-19}$ Providers often do not identify high-risk behavior for various reasons, including stigma and communication barriers..$^{19}$ Interventions to improve access to PrEP are often developed by scientists, and implemented by health care providers, who are not part of the marginalized groups they are intended to serve. ${ }^{20}$ Some studies have referenced a general lack of knowledge about PrEP among at-risk people and by some medical providers, indicating that they do not feel sufficiently trained to prescribe PrEP. Others express the need for initiatives to better educate providers on the proper indications for PrEP. Yet, there has been no study directly assessing the acceptance of and barriers to PrEP utilization among primary care residency programs as it varies between rural and nonrural areas. Therefore, we sought to assess the factors associated with PrEP prescribing in family medicine training programs and their associations on perceived PrEP competencies.

Our primary hypothesis was that nonrural family medicine residency programs would be more likely to teach PrEP versus those in more rural settings. Our second hypothesis was that programs with significant training would have more prescribing within their practices. Our third hypothesis was that programs with significant training graduate residents with greater PrEP competency.

\section{Methods}

\section{Overview and Sample}

The survey questions were part of a larger omnibus survey conducted by the Council of Academic Family Medicine Educational Research Alliance (CERA). The CERA Steering Committee evaluated questions for consistency with the overall subproject aim, readability, and existing evidence of reliability and validity. Pretesting was done on family medicine educators who were not part of the target population. Questions were modified following pretesting for flow, timing, and readability. The American Academy of Family Physicians Institutional Review Board approved the project in January 2018. Data were collected from January to February 2018.

The sampling frame for the survey was all Accreditation Council for Graduate Medical Education-accredited US family medicine residency program directors as identified by the Association of Family Medicine Residency Directors. Email invitations to participate were delivered via the online program SurveyMonkey. Six follow-up emails to encourage nonrespondents to participate were sent after the initial email invitation. There were 549 program directors at the time of the survey; 13 had previously opted out of CERA surveys.

Items included in CERA surveys are developed by workgroups who submit items within their area of inquiry for review by CERA, internal piloting, and revision under the mentorship of senior researchers. For the program director surveys, all measures were self-reported estimates provided by the program director from each residency surveyed. While no single person in a residency can speak to the behaviors and performance represented by the developed items, program directors are the stakeholder group most representative of the academic and clinical issues facing family medicine graduate medical trainees.

\section{Measures}

Initially, we planned to define programs as above or below 1 million in population. This led to a large imbalance in subgroups, as only 47 of 274 programs $(17.2 \%)$ were in areas $>1$ million. We then decided to use the US Census Bureau definition of urbanity. It uses $\geq 50,000$ as its definition of an "urbanized area." 21 The CERA omnibus survey had demarcations of 30,000 and 75,000 , so we decided to test our hypothesis using both 30,000 and 75,000 as cutoffs. One analysis compared communities $>30,000$ to those $\leq 30,000$; a second, separate analysis compared communities $>75,000$ to those $\leq 75,000$.

The level of PrEP training was used as both an independent and a dependent outcome in our analyses. We compared those with either no formal training in PrEP or some formal training (eg, as part of a lecture/ didactic) with those who identified significant training, (eg, a lecture/ didactic with routine reinforcement in clinical settings). For PrEP prescribing, we compared answers to the question "percent of patients who are appropriate for PrEP and being cared for by residents are receiving PrEP," contrasting those with $\leq 50 \%$ prescribed PrEP appropriately by residents to those with $>50 \%$ of patients. A final outcome used to explore our hypotheses included a measure of programs' self-estimation of graduating residents' level of competency in prescribing PrEP. Program directors were asked to estimate residency graduates' competency across the following categories: "None (will refer to outside provider)"; "Basic (able to screen for risk factors and overall PrEP need)"; "Independent (able to screen, order appropriate labs, and prescribe PrEP)"; or "Advanced (able to screen, order appropriate labs, prescribe PrEP, and to effectively teach others how to properly prescribe PrEP)." We compared programs reporting "none" or "basic" with programs reporting "independent" or "advanced."

We used two items to assess the largest and second-largest barriers to PrEP prescribing in residencies. Respondents were asked to identify among "Lack of resident knowledge or training," "Lack of faculty expertise," "Not enough high-risk patients," "Inadequate screening by providers," "Insufficient interest or willingness of patients," "Provider resistance to prescribing," or "Cost of medication." We calculated the proportion of respondents who identified each potential barrier as either the biggest or second-biggest barriers to 
produce an overall ranking of barriers to PrEP prescribing.

\section{Analysis}

Fisher's exact testing and expected values were calculated for assessing the association with community size, as no rural communities reported significant PrEP training for their residents. Since the numerator was 0 for that subgroup, an odds ratio calculation was not possible. For other comparison, we used logistic regression to compute odds ratios to estimate the associations between dependent and independent measures. We employed a level of statistical significance set at $P=.05$, recognizing that tests of statistical significance are approximations that serve as aids to interpretation and inference. We used Stata software (Stata Corp, College Station, TX) for the analysis.

\section{Results}

Sample

Of 549 program directors identified, 13 had previously opted out of CERA surveys, and 14 emails could not be delivered. The final sample size was therefore 522 . The overall response rate for the larger omnibus survey was $57.1 \%$ (298/522). An additional 22 programs did not complete PrEP items and were excluded from analyses leaving a final sample of 276/522 for a response rate of $52.9 \%$ for the analyzed PrEP items. Table 1 illustrates the demographics of respondents' residency programs.

A total of $15.8 \%$ of respondents reported no PrEP training in their residency and no provision of PrEP clinically for patients. Another 10.7\% of programs reported no PrEP training, but did have clinical PrEP prescribing for their patients. The majority of programs (52.0\%) reported some PrEP training in their residency with $13.8 \%$ of programs reporting significant PrEP training.

For our first hypothesis, we found that no programs training in rural communities reported significant PrEP training for their residents. Table 2 illustrates the distribution of training by community size. For our second and third hypotheses, highly significant associations were noted for the association between significant resident training in PrEP and reports of the majority of PrEP-appropriate patients receiving $\mathrm{PrEP}$ (OR=7.3; $P<.001$ ) and residents achieving independent or advanced competencies in $\mathrm{PrEP}(\mathrm{OR}=18.3$; $P<.001$ ). Table 3 illustrates the associations between reports of significant residency PrEP training and outcome measures.

Table 4 illustrates the combined proportion of barriers to PrEP prescribing. Lack of faculty expertise (23.2\%) and not enough high-risk patients $(20.5 \%)$ were the most commonly endorsed barriers to PrEP prescribing among respondents.

\section{Discussion}

The data presented illustrate a concerning proportion of training programs that include no PrEP training $(26.5 \%)$ and a significant number of programs that provide no clinical access to PrEP for their patients $(15.8 \%)$. To our knowledge, this study is the first to assess the utilization of PrEP among family medicine residency programs in rural and nonrural settings. The primary findings of our study included that residency programs that reported significant training were seven times more likely to have the majority of their PrEP-appropriate patients receiving PrEP. We also found that programs that reported significant training were 18 times more likely to graduate residents described as competent at the independent or advanced level in managing PrEP-appropriate patients. Lastly, we found that no rural family medicine programs ( $<30,000$ population) reported significant PrEP training.

HIV PrEP lies firmly within the realm of primary care and has been given a Grade A recommendation by the United States Preventive Services Task Force. ${ }^{22}$ Therefore, we sought to assess if there were geographic disparities among family medicine residencies regarding their acceptance of and barriers to PrEP utilization. Our survey respondents had a large distribution across geographic regions and community size, with the greatest percentage coming from community based, universityaffiliated residency programs. These community-level gaps in residency PrEP training risk producing family medicine physicians who are unprepared to offer the full range of HIV prevention options to appropriate patients. This may ultimately contribute to avoidable HIV infections.

While we did not have a specific hypothesis about the most likely barrier to PrEP prescribing, we found that faculty expertise and having enough high-risk patients were the most likely to be the top choice of respondents. These data fit with existing literature describing how a lack of provider knowledge about PrEP leads to discomfort prescribing it. ${ }^{23,24}$ Current literature also shows regional disparities in PrEP prescribing, which is relatively low in the South/Southeastern United States. ${ }^{25,26}$ HIV disproportionately affects those living in the Southeast United States, yet this is also a region where PrEP is prescribed with relatively low frequency. ${ }^{26}$ While the disparity between regions with high HIV prevalence and relatively low PrEP prescribing is multifactorial, it begs the question: are providers truly taking sexual histories that assess for HIV risk behaviors? If there is a lack of faculty expertise regarding PrEP, patients are less likely to be accurately screened for PrEP-appropriateness. This could, in turn, contribute to the misperception of lacking enough high-risk patients. Each of these barriers potentially drives lower levels of training for family medicine residents, perpetuating an unpreparedness to prescribe PrEP.

The limitations of this study include the limits of self-reported data and biases related to social desirability. For example, program directors identifying prescribing rates to eligible patients may overestimate this variable if their clinic underscreens 
Table 1: Description of Sample

\begin{tabular}{|c|c|c|}
\hline Characteristic & $\mathbf{n}$ & $\%$ \\
\hline \multicolumn{3}{|c|}{ Type of Residency Program } \\
\hline University based & 47 & 17.0 \\
\hline Community based, university affiliated & 166 & 60.1 \\
\hline Community based, nonaffiliated & 48 & 17.4 \\
\hline Military & 8 & 2.9 \\
\hline Other & 7 & 2.5 \\
\hline \multicolumn{3}{|c|}{ Geographic Region } \\
\hline New England (NH, MA, ME, VT, RI, CT) & 11 & 4.0 \\
\hline Middle Atlantic (NY, PA, NJ) & 38 & 13.8 \\
\hline South Atlantic (PR, FL, GA, SC, NC, VA, DC, WV, DE, MD) & 34 & 12.3 \\
\hline East South Central (KY, TN, MS, AL) & 12 & 4.4 \\
\hline East North Central (WI, MI, OH, IN, IL) & 53 & 19.2 \\
\hline West South Central (OK, AR, LA, TX) & 28 & 10.1 \\
\hline West North Central (ND, MN, SD, IA, NE, KS, or MO) & 28 & 10.1 \\
\hline Mountain (MT, ID, WY, NV, UT, AZ, CO, NM) & 24 & 8.7 \\
\hline Pacific (WA, OR, CA, AK, HI) & 48 & 17.4 \\
\hline \multicolumn{3}{|c|}{ Community Size } \\
\hline Less than 30,000 & 27 & 9.9 \\
\hline 30,000 to 74,999 & 51 & 18.6 \\
\hline 75,000 to 149,000 & 45 & 16.4 \\
\hline 150,000 to 499,999 & 63 & 23.0 \\
\hline 500,000 to 1 million & 41 & 15.0 \\
\hline$>1$ million & 47 & 17.2 \\
\hline \multicolumn{3}{|c|}{ Number of Residents } \\
\hline$<19$ & 99 & 36.1 \\
\hline $19-31$ & 132 & 48.2 \\
\hline$>31$ & 43 & 15.7 \\
\hline
\end{tabular}

for appropriate patients. While program directors are the stakeholder group best able to respond to the breadth of items included in the survey, there are limitations to the ability of respondents to provide valid estimates of resident competencies and clinical delivery of PrEP services. Given the impact of social desirability, we assume that our results are conservative estimates of the educational challenges related to PrEP training illustrated by our data. While the survey had a reasonable response rate, it is always possible that nonresponders would have been systematically different from responders resulting in bias. While we would like to make inferences for primary care, it is very possible and even likely that family medicine training programs may be different from other primary care training professions. While we believe that our community size comparisons are meaningful, the limits of the available survey response options did not allow us to map standard community size measures for rural versus urban comparisons. Finally, cross-sectional data can only suggest associations, and we must be cautious in making causal assumptions from our findings.

Potential next steps include an improved delineation of best practices for training future primary care providers in the assessment of HIV risk and the use of PrEP. In addition to developing and augmenting existing training curricula, continuing medical education venues will 
Table 2: Distribution of Significant PrEP Training and Community Size

\begin{tabular}{|c|c|c|c|c|}
\hline & & \multicolumn{2}{|c|}{ Community Size $<30 \mathrm{~K}$} & \multirow{2}{*}{ Fisher's Exact $P$ Value } \\
\hline & & No & Yes & \\
\hline \multirow{2}{*}{ Significant PrEP Training } & No & 205 (209.1)a & $27(22.9)$ & \multirow{2}{*}{.019} \\
\hline & Yes & $41(36.9)$ & $0(4.1)$ & \\
\hline & & \multicolumn{2}{|c|}{ Community Size $<75 \mathrm{~K}$} & \multirow{2}{*}{ Fisher's Exact $P$ Value } \\
\hline & & No & Yes & \\
\hline \multirow{2}{*}{ Significant PrEP Training } & No & $162(165.7)$ & $70(66.3)$ & \multirow{2}{*}{.192} \\
\hline & Yes & $33(29.3)$ & $8(11.7)$ & \\
\hline
\end{tabular}

Abbreviation: PrEP, Pre-exposure prophylaxis.

a Expected value using Stata "expected" command.

Bold font indicates statistical significance $(P<.05)$.

Table 3: Associations With Significant PrEP Training

\begin{tabular}{|l|c|c|}
\hline \multicolumn{1}{|c|}{ Associations With Training } & \multicolumn{2}{c|}{ Significant Training } \\
\cline { 2 - 3 } & Odds Ratio & $\boldsymbol{P}$ Value \\
\hline $\begin{array}{l}\text { >50\% of PrEP-appropriate patients receiving PrEP } \\
\text { Reference: } \leq 50 \% \text { of PrEP-appropriate patients receiving PrEP }\end{array}$ & 7.27 \\
\hline $\begin{array}{l}\text { Independent-to-advanced PrEP competency } \\
\text { Reference: none-to-basic competency }\end{array}$ & 18.33 & $<.001$ \\
\hline
\end{tabular}

Abbreviation: PrEP, preexposure prophylaxis.

Bold font indicates statistical significance $(P<.05)$.

Table 4: Barriers to PrEP (Each Respondent Could Choose Two)

\begin{tabular}{|l|c|}
\hline & $\begin{array}{c}\text { Top Barriers to PrEP Identified } \\
\text { n (\%) }\end{array}$ \\
\hline Faculty expertise & $138(23.2)$ \\
\hline Enough high-risk patients & $122(20.5)$ \\
\hline Inadequate screening & $87(14.6)$ \\
\hline Resident knowledge/training & $74(12.4)$ \\
\hline Patient interest & $56(9.4)$ \\
\hline Medication costs & $55(9.2)$ \\
\hline Provider resistance & $10(1.7)$ \\
\hline
\end{tabular}

Abbreviation: PrEP, preexposure prophylaxis.

need to adopt similar training priorities in order to enhance spread and uptake among already-practicing providers. Providers should be encouraged to work as part of interprofessional teams, including care navigators, pharmacists, and other health care providers as resources. At-risk populations should be empowered to ask for PrEP, and tools should be developed along with these populations for ways to bridge communication gaps and reduce stigma. Additionally, in clinical settings with lower-risk populations, residents could be encouraged to participate in rotations with higher-risk populations to increase their exposure to appropriate PrEP prescribing.
In conclusion, we found that rural family medicine residency programs lack PrEP training and programs that provide significant PrEP training are more likely to prescribe it to appropriate patients as well as graduate residents who are prepared to do the same. HIV is a national epidemic, and requires a national response. The COVID-19 pandemic 
requires us to rededicate ourselves to prevention of disease transmission, recognizing that prevention is the most powerful tool in controlling epidemics. PrEP has the potential to impact the incidence of new HIV infections significantly, which disproportionately affect young MSM of color and transgender women of color. As such, PrEP prescribing is a tool of social justice. In partnership with patient populations they are intended to serve, family medicine residencies must commit themselves to improving PrEP uptake and adherence. Family physicians are at the frontlines of preventative health throughout the country and, given the appropriate training, have the power to curb rising rates of HIV infections among marginalized and vulnerable populations, and expand the reach of PrEP overall.

FINANCIAL SUPPORT: No specific funding sources supported this work. Neither this study as a whole, nor any role in its design including collection, analysis, and interpretation of data, report writing, or the decision to submit the report for publication, received any sponsorship.

PRESENTATIONS: This study was presented as a completed research project presented at the 2019 Society of Teachers of Family Medicine Annual Spring Conference, in Toronto, Ontario, Canada.

CORRESPONDING AUTHOR: Address correspondence to Dr Byron K. Jasper, Byja Health Systems, Inc., 9655 Perkins Road C-237, Baton Rouge, LA 70810. 225-320-5090. byron.jasper@cmmpmed.org.

\section{References}

1. Thigpen MC, Kebaabetswe PM, Paxton LA, et al; TDF2 Study Group. Antiretroviral preexposure prophylaxis for heterosexual HIV transmission in Botswana. N Engl $\mathrm{J}$ Med. 2012;367(5):423-434. doi:10.1056/NEJMoa1110711

2. Baeten JM, Donnell D, Ndase P, et al; Partners PrEP Study Team. Antiretroviral prophylaxis for HIV prevention in heterosexual men and women. N Engl J Med. 2012;367(5):399-410. doi:10.1056/NEJMoa1108524

3. Grant RM, Lama JR, Anderson PL, et al; iPrEx Study Team. Preexposure chemoprophylaxis for HIV prevention in men who have sex with men. N Engl J Med. 2010;363(27):2587-2599. doi:10.1056/NEJMoa1011205

4. Molina J, Capitant C, Spire B, et al. On demand PrEP with oral TDF-FTC in MSM: results of the ANRS Ipergay trial. Conference on Retroviruses and Opportunistic Infections. Seattle, 2015.
5. McCormack S, Dunn DT, Desai M, et al. Preexposure prophylaxis to prevent the acquisition of HIV-1 infection (PROUD): effectiveness results from the pilot phase of a pragmatic open-label randomized trial. Lancet. 2015.

6. Donnell D, Baeten JM, Bumpus NN, et al. HIV protective efficacy and correlates of tenofovir blood concentrations in a clinical trial of PrEP for HIV prevention. J Acquir Immune Defic Syndr. 2014;66(3):340-348. doi:10.1097/ QAI.0000000000000172

7. Grant RM, Anderson PL, McMahan V, et al; iPrEx study team. Uptake of pre-exposure prophylaxis, sexual practices, and HIV incidence in men and transgender women who have sex with men: a cohort study. Lancet Infect Dis. 2014;14(9):820-829. doi:10.1016/S14733099(14)70847-3

8. Anderson PL, Glidden DV, Liu A, et al; iPrEx Study Team. Emtricitabine-tenofovir concentrations and pre-exposure prophylaxis efficacy in men who have sex with men. Sci Transl Med. 2012;4(151):151ra125. doi:10.1126/scitranslmed.3004006

9. Siegler AJ, Bratcher A, Weiss KM. Geographic Access to Preexposure Prophylaxis Clinics Among Men Who Have Sex With Men in the United States. Am J Public Health. 2019;109(9):1216-1223. doi:10.2105/ AJPH.2019.305172

10. National Advisory Committee on Rural Health \& Human Services. May 2020, HIV Prevention and Treatment Challenges in Rural America: Policy Brief and Recommendations to the Secretary. Washington, DC: Department of Health and Human Services; May 2020. Accessed September 2, 2021. https://www.hrsa.gov/sites/ default/files/hrsa/advisory-committees/rural/ publications/2020-rural-hiv-prev-treat-call.pdf

11. Karris MY, Beekmann SE, Mehta SR, Anderson CM, Polgreen PM. Are we prepped for preexposure prophylaxis (PrEP)? Provider opinions on the real-world use of PrEP in the United States and Canada. Clin Infect Dis. 2014;58(5):704-712. doi:10.1093/cid/cit796

12. Desai M, Gafos M, Dolling D, McCormack S, Nardone A. Healthcare providers' knowledge of, attitudes to and practice of pre-exposure prophylaxis for HIV infection. HIV Med. 2015

13. Krakower DS, Oldenburg CE, Mitty JA, et al. Knowledge, Beliefs and Practices Regarding Antiretroviral Medications for HIV Prevention: Results from a Survey of Healthcare Providers in New England. PLoS One. 2015;10(7):e0132398. doi:10.1371/journal. pone.0132398

14. Desai M, Gafos M, Dolling D, McCormack S, Nardone A; PROUD study. Healthcare providers' knowledge of, attitudes to and practice of pre-exposure prophylaxis for HIV infection. HIV Med. 2016;17(2):133-142. doi:10.1111/ hiv.12285

15. Arnold EA, Hazelton P, Lane T, et al. A qualitative study of provider thoughts on implementing pre-exposure prophylaxis (PrEP) in clinical settings to prevent HIV infection. PLoS One. 2012;7(7):e40603. doi:10.1371/journal. pone. 0040603
16. Tellalian D, Maznavi K, Bredeek UF, Hardy WD. Pre-exposure prophylaxis (PrEP) for HIV infection: results of a survey of HIV healthcare providers evaluating their knowledge, attitudes, and prescribing practices. AIDS Patient Care STDS. 2013;27(10):553-559. doi:10.1089/ apc. 2013.0173

17. White JM, Mimiaga MJ, Krakower DS, Mayer KH. Evolution of Massachusetts physician attitudes, knowledge, and experience regarding the use of antiretrovirals for HIV prevention. AIDS Patient Care STDS. 2012;26(7):395-405. doi:10.1089/apc.2012.0030

18. Tripathi A, Whiteside YO, Duffus WA. Perceptions and attitudes about preexposure prophylaxis among seronegative partners and the potential of sexual disinhibition. South Med J. 2013;106(10):558-564. doi:10.1097/ SMJ.0000000000000010

19. Harkness A, Bainter SA, Mendez NA, et al. Factors associated with barriers to engagement in HIV-prevention care among sexual minority men. J Behav Med. 2021. doi:10.1007/s10865021-00230-0

20. Sutton MY, Martinez O, Brawner BM, et al. Vital voices: HIV prevention and care interventions developed for disproportionately affected communities by historically underrepresented, early-career scientists. J Racial Ethn Health Disparities. 2020. doi:10.1007/s40615-02000908-2

21. Urban and Rural. United States Census Bureau. Revised June 16, 2021. Accessed March 29, 2021. https://www.census.gov/programssurveys/geography/guidance/geo-areas/urbanrural.html Accessed: March 29, 2020.

22. Owens DK, Davidson KW, Krist AH, et al; US Preventive Services Task Force. Preexposure Prophylaxis for the Prevention of HIV Infection: US Preventive Services Task Force Recommendation Statement. JAMA. 2019;321(22):2203-2213. doi:10.1001/ jama.2019.6390

23. Pleuhs B, Quinn KG, Walsh JL, Petroll AE, John SA. Health care provider barriers to HIV pre-exposure prophylaxis in the United States: a systematic review. AIDS Patient Care STDS. 2020;34(3):111-123. doi:10.1089/apc.2019.0189

24. Turner L, Roepke A, Wardell E, Teitelman AM. Do You PrEP? A review of primary care provider knowledge of $\mathrm{PrEP}$ and attitudes on prescribing PrEP. J Assoc Nurses AIDS Care: JANAC. 2018;29(1):83-92. doi:10.1016/j. jana.2017.11.002

25. Zhang C, McMahon J, Fiscella K, et al. HIV Pre-exposure prophylaxis implementation cascade among health care professionals in the United States: implications from a systematic review and meta-analysis. AIDS Patient Care STDS. 2019;33(12):507-527. doi:10.1089/ apc.2019.0119

26. Henny KD, Duke CC, Buchacz K, Brooks JT, Samandari T, Sutton MY. HIV prescriptions on the frontlines: primary care providers' use of antiretrovirals for prevention in the Southeast United States, 2017. Prev Med. 2020;130:105875. doi:10.1016/j. ypmed.2019.105875 